\title{
EFFICIENT POINT CLOUD COLLISION DETECTION AND ANALYSIS IN A TUNNEL ENVIRONMENT USING KINEMATIC LASER SCANNING AND K-D TREE SEARCH
}

\author{
Johannes Schauer $^{\mathrm{a}}$, Andreas Nüchter ${ }^{\mathrm{b}}$ \\ ${ }^{a}$ School of Engineering and Science, \\ Automation Group, Jacobs University of Bremen $\mathrm{gGmbH}$, \\ Campus Ring 1, Bremen 28759, Germany \\ j.schauer@jacobs-university.de \\ ${ }^{\mathrm{b}}$ Informatics VII : Robotics and Telematics, Julius-Maximilians-University Würzburg, \\ Am Hubland, Würzburg 97074, Germany \\ andreas@nuechti.de, http://www. nuechti.de
}

KEY WORDS: Collision Detection, K-D Tree, Kinematic Laser Scanning, 3D Point Clouds

\begin{abstract}
:
Measuring the structure gauge of tunnels and other narrow passages has so far been the only way to evaluate whether large vehicles can pass through them. But especially for very long vehicles like train wagons and their cargo, the structure gauge is an insufficient measure because the center part of the vehicle between two bogies will inevitably leave the swept volume of its cross section when moving along any other trajectory than a straight line perpendicular to its cross section. In addition, the vehicle as well as the cargo must keep a minimum safety margin from the environment at all points of its trajectory. This paper explores an automated method to check for possible collisions of a model represented by a 3D point cloud moving through the 3D point cloud of an environment. We were given environment data of a train track through a narrow tunnel where simply relying on the structure gauge would indicate that a given wagon would pass through without any collision even though in reality, the train wagon would collide with the inner tunnel wall inside a sharp turn of the tracks. The k-d tree based collision detection method presented in this paper is able to correctly highlight these collisions and indicate the penetration depth of each colliding point of the environment into the model of the train wagon. It can be generalized for any setup where two static point clouds have to be tested for intersection along a trajectory.
\end{abstract}

\section{INTRODUCTION AND PROBLEM FORMULATION}

The minimum clearance outline or structure gauge has an important place in the planning of railroad tracks. It is the swept volume of the minimum cross section that must be kept free of any obstacles. A similar measure is the loading gauge which is the swept volume of the cross section of a train wagon moved along a track. The difference between the two is the engineering tolerance or clearance. The structure gauges along a track together with the maximum loading gauge determine whether or not a train with certain cargo can go along a given route or how much space around new tracks has to be kept clear and is subject to a number of decades old standards and regulations (Siegmann, 2011).

Unfortunately the structure gauge is an unsatisfying measure within turns of the tracks. Figure 1 visualizes the problem. The curvature represents the loading gauge of the train wagon in gray. The dark gray areas represent the volume of the train wagon which is outside of its loading gauge during the turn.

The algorithm that will be presented in the following requires three objects as input: the pointcloud of the environment which was collected by driving a Optech Lynx Mobile Mapper along the train tracks but can also be acquired using the methods presented in (Elseberg et al., 2014). The second input is a point cloud of the model which was acquired by taking seven scans of a real train wagon with a Riegl VZ-400 laser scanner and then registering them using 3DTK (Nüchter et al., 2010). The third input is the trajectory of the train tracks.

The goal is to determine which points of the environment collide with the model on its path, given a certain safety margin (the minimal allowed clearance) and how deep any colliding points penetrate the model. To this end a k-d tree of the environment was

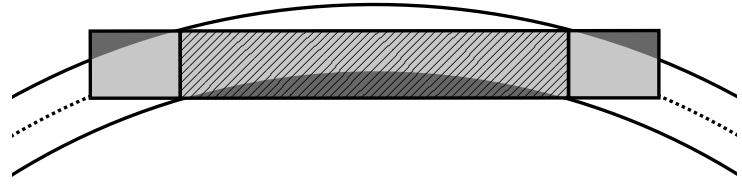

Figure 1: Top view of the train wagon (in dark and light gray) and its curved loading gauge as it passes through a turn. The dark gray areas mark the volumes of the train wagon outside of its loading gauge. The striped volume indicates the volume of the train wagon between its two bogies. The dotted line indicates the wagon's trajectory.

created, the model was moved through it along its trajectory and a k-d tree search was performed around the points of the model to find colliding points and their penetration distance.

The contributions of this paper can be summarized as:

- a method to perform easily parallelizable collision detection of a single arbitrary (and deformable) point cloud (the model) with a static environment in two variants

- two easily parallelizable methods to calculate penetration depth of the model with the environment

- a highly optimized k-d tree implementation and query functions to perform collision detection

A right handed coordinate system will be assumed. With reference to the train wagon, the $z$-axis will be the up-vector and the train wagon will be moved along the $y$-axis. The coordinate system of the train wagon has its origin in its center of mass. This is important for calculating rotations and penetration depths. 
The rest of the paper is organized as follows: The next section covers related work to the one presented in this paper. Section three presents the k-d tree data structures and algorithms. Section four and five present our methods for collision detection and penetration depth calculation using the k-d tree, respectively. Section six show benchmark results while section seven concludes this paper.

\section{RELATED WORK}

Collision detection, which is also called interference detection or intersection searching, is a well studied topic in computer graphics (Jiménez et al., 2001, Lin and Gottschalk, 1998) because of its importance for dynamic computer animation and virtual reality applications (Tzafestas and Coiffet, 1996). On the other hand, their work is limited to collision detection between geometric shapes and polygonal meshes whereas most sensor data is acquired as point clouds. While collision detection is also relevant for motion planning in the field of robotics, it is a less studied problem there. Collision detection between point clouds was for example researched by Klein and Zachmann (Klein and Zachmann, 2004) who use the implicit surface created by a point cloud to calculate intersections. Another example is the recent work by Hermann et al. (Hermann et al., 2014) who use voxels to check for spatial occupancy for robot motion planning.

Existing techniques make use of very similar approaches. One method is to apply a spatial hierarchical partitioning of the input geometry using octrees (Jung and Gupta, 1996), BSP-trees (Ar et al., 2000) or k-d trees (Held et al., 1995) . Other solutions apply regular partitioning using voxels (Garcia-Alonso et al., 1994, Hermann et al., 2014). The goal of any partitioning is to be able to quickly search and check only the relevant geometries in the same or neighboring cells. The method presented in this paper will make use of a hierarchical $\mathrm{k}-\mathrm{d}$ tree for the environment in combination with a regular partitioning of the model into a grid of bounding spheres.

Another method is to use hierarchies of bounding volumes like spheres (Hubbard, 1996), axis aligned bounding boxes, oriented bounding boxes (Gottschalk et al., 1996) or discrete oriented polytopes (Klosowski et al., 1998). Optimizing the regular grid that was generated for the model into a hierarchical structure will be left for future work.

Collision detection methods can be divided in those for static and deformable objects (Teschner et al., 2003, Bergen, 1997). While the method presented in this paper does not easily allow changes in the environment because it would require a recalculation of its $\mathrm{k}-\mathrm{d}$ tree, arbitrary changes in the point cloud of the model are possible without any performance impacts.

Another classification is whether the algorithm easily allows multiple moving objects. Using a brute-force approach such algorithms would have a runtime of $O\left(n^{2}\right)$ for $n$ objects because every possible pair of objects is checked for collisions. Modern approaches like the I-COLLIDE system (Cohen et al., 1995) use a "sweep and prune" approach to minimize the amount of necessary checks. Another approach is to dynamically adjust the search tree to account for object movements (Luque et al., 2005). The method in this paper does not handle multiple moving models.

Calculating the penetration depth of one object into another is important to calculate the force of collisions and respond accordingly in virtual reality applications (Tzafestas and Coiffet, 1996). It is also important for visualization purposes, to differently highlight objects reaching into a safety margin with an indication of how much they violate the constraint. This application was shown in prior work on this topic by the authors of this pa-

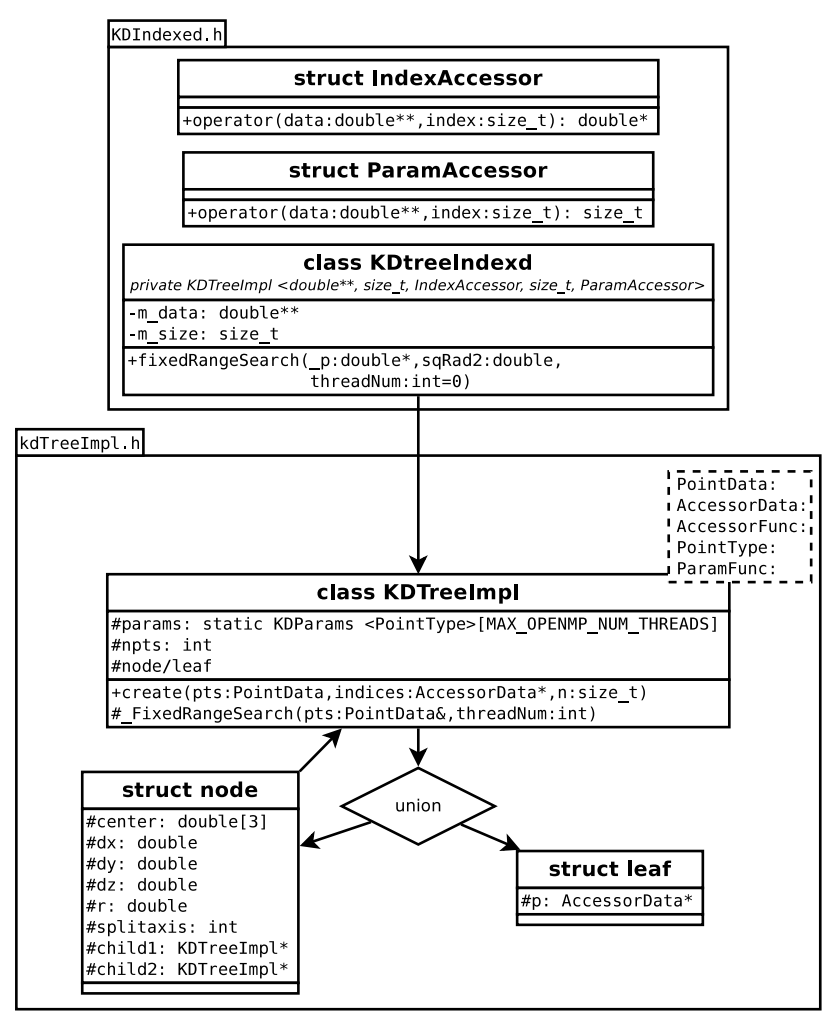

Figure 2: Boxes are in UML, relationships (arrows) are not. KDTreeImpl is templated by KDtreeIndexed with the parameters listed in the comment. The node and leaf structure are a $\mathrm{C}++$ union. The params member of KDTreeImpl is static. UML packages are used to indicate file membership and to group for readability. Only the fixedRangeSearch search function and its recursive counterpart _FixedRangeSearch are listed for brevity.

per (Elseberg et al., 2014).

\section{DATA STRUCTURES FOR EFFICIENT COLLISION DETECTION AND DEPTH OF PENETRATION CALCULATION}

In this section our highly-optimized k-d tree implementation is presented. It is implemented in 3DTK in C++. It currently implements multiple search functions, can be parameterized to be used with $3 \mathrm{D}$ point data of different precision and container type, allows to present search results as pointers, array indices or as 3D coordinate data and allows parallel execution through OpenMP.

Tree data structure Consider the object relationship sketched in figure 2. Each node in the k-d tree stores an integer npts indicating the number of points this node contains. If this value is non-zero, the node is a leaf node. Otherwise, the node is an inner node. A union structure stores data depending on the node type. Inner nodes store their center coordinate, the node size, the coordinate axis by which the node is split and pointers to the two children the node is split into. Leaf nodes store a pointer $\mathrm{p}$ to an array representing the contained points. The static member params of type KDParams stores information associated with the current k-d tree search. As a static member, this container is only stored in memory once.

Creation of a k-d tree A k-d tree is created by calling a node's create function with the points to fill the tree with. If This function gets passed less than 10 points, then a leaf node is created. Otherwise the axis aligned bounding box of the passed data is calculated and stored, an axis aligned plane to split the data is found 
by finding the longest axis of the bounding box and the data is partitioned into the new two child nodes. The function create is then called recursively with the points falling into each subtree, respectively.

Searching the k-d tree Spacial search in point clouds can be parameterized by two properties: the location (where to search for results) and the subject (what to return). The following five search areas are implemented by 3DTK:

(a) radius $r$ around a point $P_{1}$

(b) radius $r$ around an infinite line defined by a point $P_{1}$ and a direction vector $v$

(c) radius $r$ around an infinite ray defined by $P_{1}$ and $v$

(d) radius $r$ along a finite line segment defined by points $P_{1}$ and $P_{2}$ and

(e) inside an axis aligned bounding box defined by $P_{1}$ and $P_{2}$ as the corners with minimum and maximum coordinate values, respectively

Additional search volumes that could be added in the future would be oriented bounding boxes, cylinders or general polytopes. In most volumes, searches for the following result types can be performed:

(1) the point closest to $P_{1}$

(2) the $k$ points closest to $P_{1}$

(3) all points within the search volume

(4) the point closest to the given line, ray or line segment

(5) the $k$ closest points to the given line, ray or line segment

After eliminating the inapplicable combinations, one ends up with 19 meaningful search functions. A full list is omitted for brevity. For example, the common nearest-neighbor search (NNS) would be searching for the closest point to $P_{1}(1)$ in a radius $r$ around a point $P_{1}$ (a). For the collision detection method presented in this paper, the following four functions are needed:

- FindClosest: closest point to a coordinate: (a) and (1)

- fixedRangeSearch all points around a coordinate: (a) and (3)

- segmentSearch_1NearestPoint closest point to a line segment: (d) and (1)

- segmentSearch_all all points around a line segment: (d) and (3)

fixedRangeSearch The general operation of the search functions will be presented by using the function $f$ ixedRangeSearch as an example. The functions is implemented in the class KDTreeIndexed. It sets up the KDParams structure with the search parameters and then calls the recursive function FixedRangeSearch (notice the leading underscore) implemented in KDTreeImpl.

All recursive search functions can be divided into three functional parts. Firstly, if the node is a leaf node, then all points the node contains are checked for satisfiability of the search criteria. Secondly, if the node is an inner node, then a check is done whether the node can possibly contain parts of the result. If not, the function returns. Otherwise, thirdly, the search recurses into one or both child nodes.
The recursive function _FixedRangeSearch returns all points within a radius $r$ around a point $P$. The satisfiability check for leaf nodes involves checking the distance criteria of radius $r$ around $P$ and pushing the point to the result vector if its distance from $P$ is less than $r$. The parameterized functions of type IndexAccessor and ParamAccessor are used to return coordinate data or data of the return type for each point in the leaf node, respectively. They do not pose a performance overhead as they are usually inlined by the compiler.

To check whether to abort, the following fast heuristic is used. Suppose an intermediate node inside the k-d tree with center coordinate $C$ and a bounding box size of $d_{x}, d_{y}$ and $d_{z}$. Suppose the six sides of the node's axis aligned bounding box form six axis aligned planes: each plane being the infinite extension of the six sides of the node's bounding box. Opposing sides of the node's bounding form pairs of parallel planes. Three of these plane pairs are created, one pair along each dimension. Then the distance of $P$ to the closest plane of each pair of planes is found. If $P$ is between a pair of planes, then its distance is represented as a negative value. Then the maximum distance of the resulting three distance values is taken (one for each dimension). If the maximum value $d_{P}$ is negative, then all three coordinate values of $P$ must lie inside the current node's bounding box and the search has to recurse into one or both child nodes. If the maximum value is positive and larger than the search distance, then the current node cannot contain any results and the function returns without recursing deeper into the tree. While the geometry behind this check seems complex, the calculations can be done with very few and inexpensive operations thanks to the axis alignment of the bounding box (see equation 1).

$$
\begin{gathered}
d_{P}=\max \left(\max \left(\left|P_{x}-C_{x}\right|-d_{x},\left|P_{y}-C_{y}\right|-d_{y}\right),\right. \\
\left.\left|P_{z}-C_{z}\right|-d_{z}\right)
\end{gathered}
$$

The last part of each search function recurses into the child nodes. First, a check for the point's position relative to the split axis of the current node decides which child node to recurse fist. Whether or not the second child node is recursed into as well depends on whether the bounding cube of the search radius around $P$ extends into the second child of the current node as well or not.

\section{COLLISION DETECTION}

Two variants of collision detection were implemented using the $\mathrm{k}$-d tree. One variant is based on a range search around each point of the model using FixedRangeSearch and the other is based on a segment search between two subsequent points of the model on its trajectory using segmentSearch_all. In both variants, the model is moved along its trajectory and a range or segment k-d tree search with radius $r$ is performed at each position. When points are found to be colliding, then this information is saved in a separate boolean vector which stores for each point in the environment whether it ever collided with the model on its trajectory or not. The search radius $r$ determines the precision of both algorithms. The smaller the search radius, the more precise the collision detection is. For smaller search radii, the model has to be sampled dense enough to not leave any unoccupied volume. The search radius $r$ is the required "safety distance" between the model and the environment within which no point of the environment should lie. At the end, the collision information from the boolean vector is used to partition the environment into colliding and non-colliding points.

Both variants are embarrassingly parallel operations. All k-d tree searches can be run in parallel and even updating of the boolean collision vector can be done in parallel as its values are only ever 
written but not read during collision detection.

Range search In this variant, on each position of the model on its trajectory, a fixed range search using FixedRangeSearch is done around each point of the model. All points of the environment that are found to be within range $r$ of any point of the model at any position on its trajectory are updated to be colliding.

Segment search This variant executes a segment search using segmentSearch_all between the same points of the model at two subsequent positions of the model in the environment. This means that in this variant, trajectory positions can be further apart than the search radius because the space between two positions is searched by linearly connecting the two. In effect, this variant can thus require less search operations while maintaining a similar result quality compared to the range search.

\section{DEPTH OF PENETRATION CALCULATION}

Two variants to calculate depth of penetration will be presented. They perform differently depending on the kind of input data and yield different results depending on the sampling rate of the model trajectory. The first variant is generally faster but produces only good results for objects protruding the path of the model through the environment. The first variant does not produce correct results when the model moves alongside a will and collides with it.

The first variant is an embarrassingly parallel operation just as the collision detection methods. The second variant easy to parallelize as well and the only part of the second variant that has to be synchronized between workers is the updating of the penetration depth because it requires reading and checking the already stored depth of penetration per colliding point.

Heuristic for protruding objects This variant iterates through all points of the environment that were found to be colliding and finds the closest non-colliding point using FindClosest. The distance between the two points is then recorded as the depth of penetration. This variant works well for objects that "stick" into the path of the model because the penetration depth of the tip of that object will be about as deep as its distance to the closest non-colliding point. This method was shown to work well for automotive assembly lines as shown in prior work of the authors (Elseberg et al., 2014).

General depth of penetration calculation Consider figure $3 \mathrm{a}$ which illustrates this method. Figure 3a shows a top view of the train wagon model at one point of its trajectory inside the tunnel. It is shown colliding with the right hand side tunnel wall. The algorithm iterates over every point of the model $P_{n}$ and finds its projection to the wagon center $A_{n}$. Since the central axis is the $y$-axis in the coordinate system of the train wagon, this projection is simply done by setting the $x$ and $z$ coordinates to zero. Then a segment search using segmentSearch_1NearestPoint on the line segment from $P_{n}$ to $A_{n}$ is performed for every point of the model: for each point $P_{n}$ the closest point $C_{n}$ of the colliding environment within the search radius is found. A fixed range search using FixedRangeSearch of radius $r$ around $C_{n}$ is performed and all points within that search radius including $C_{n}$ are collected. This collection of points has to be performed because otherwise, many points of the environment would be missed by segmentSearch_1NearestPoint. The distance between $C_{n}$ and $P_{n}$ is calculated and that distance is assigned to all points that were found by FixedRangeSearch if the new distance value is greater than the old one. This set of calculations is done for each point of the model on each position of its trajectory. In the end, every colliding point of the environment has attached to it the greatest distance found by this method over the whole trajectory.

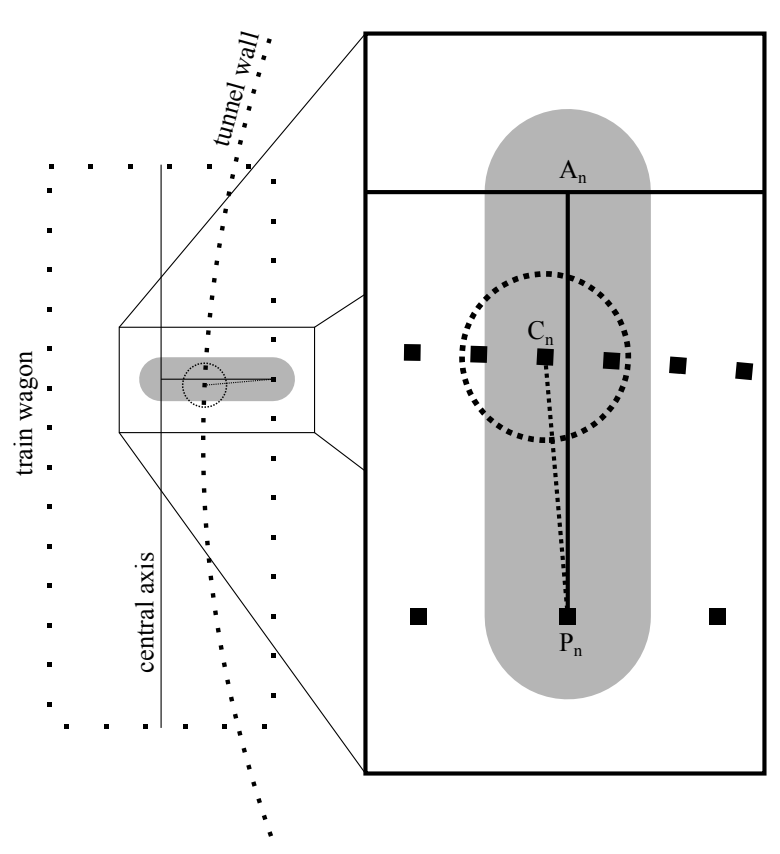

(a) Left: a top view of the train wagon at a position through the tunnel. Right: a magnified and rotated part of the left figure with point names. The gray area represents the segment search volume. The dotted line is the distance between $P_{n}$ and $C_{n}$ which is the point that was found to be closest to $P_{n}$ within the search area. The dotted circle shows the search radius around $C_{n}$. All points within this radius are updated with the same distance that $C_{n}$ has to $P_{n}$ if that distance is greater than the previously stored one.

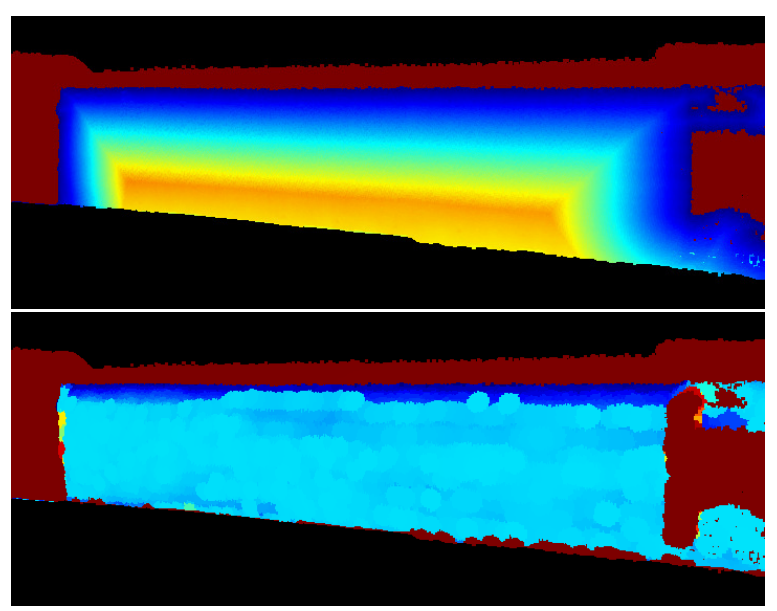

(b) A comparison of the penetration depth as calculated by the two variants. Both figures show a narrow piece of tunnel from the outside with the calculated penetration depth indicated by the point color. Non-colliding points are shown in dark red. The top figure shows the simple and fast variant while the bottom figure shows the more correct slower variant.

Figure 3: Depth of penetration calculation 
This method requires that the individual points of the trajectory are not further apart than the search radius. While this is also one of the reasons why this method is more computationally expensive than the first heuristic, it also yields better results when applied to a collision with the tunnel wall. Figure $3 b$ illustrates the difference.

\section{EXPERIMENTS AND RESULTS}

A 3D point cloud of train tunnel was provided to us by TopScan $\mathrm{GmbH}$. The point cloud contains 18.92 million points of outdoor data. The point cloud was collected by a Optech Lynx Mobile Mapper mounted on a van which was placed on a train wagon (see figure 4a). TopScan also provided the trajectory data to us which was comprised of 23274 positions over a distance of $1144 \mathrm{~m}$. The trajectory contains positional as well as orientation data.

To retrieve a point cloud of a suitable model to move through the environment, a train wagon was manually scanned using a RIEGL VZ-400 laser scanner. Seven scans were taken from all sides of the wagon and registered using 3DTK's SLAM implementation. The train wagon was manually extracted from the resulting registered point cloud by using 3DTK's show application. It was then aligened inside the axis aligned bounding box of the wagon. The alignment process can be seen in figure 4c. As calibration data of the precise location of the scanner relative to the environment was missing, our results can only serve a demonstration purpose of our methods. The final point cloud of the wagon contained 2.5 million points.

The trajectory that was provided to the authors included orientation information in three degrees of freedom as well. Since a train wagon is mounted on two bogies and since the origin of the coordinate system of the train is located in its center, using this trajectory directly would've meant that the wagon would rotate around its own center along the trajectory. This would produce wrong results since instead, the bogies of the train have to remain on the tracks while the center follows accordingly. A new trajectory was calculated from the original trajectory by assuming a bogie distance of $20 \mathrm{~m}$ and moving the train wagon such that the center of both bogies would always be on the original trajectory. Since this operation requires the original trajectory to be a continuous function and not a sampled trajectory, a spline was fitted across all points of the trajectory with a sum of squared residuals over all the control points of $10 \mathrm{~m}$. This amounts to the spline only a few millimeter away on average from the original trajectory. The FITPACK library (Dierckx, 1993) was used to calculate the spline. The result of this computation also adjusted the yaw and pitch of the trajectory.

To benchmark the developed algorithms, the train wagon model as well as the trajectory were sampled with several different point distances. For the train wagon, the original amount of 2.5 million points was reduced using 3DTK's scan_red program which allows an octree based reduction of a point cloud with a given voxel size. As the search volume for collision detection must not contain any holes,p a model of equidistant points was created by saving the center of each occupied octree voxel as point of the reduced model. This creates a 3D square lattice of points. Five different reductions of the train wagon point cloud were created to run benchmarks on them. Due to the structure of the underlying octree, the voxel size $d_{m}$ was repeatedly halved starting from a maximum voxel size of $0.924 \mathrm{~m}$ and down to a voxel size of $5.8 \mathrm{~cm}$. For each of the five reductions, the search radius was chosen to create a bounding sphere of an octree voxel of the respective size. That way, all space occupied by the model is searched for collisions without leaving any holes. This means that the voxel size $d_{m}$ computes from the bounding sphere and search radius $r$ as $d_{m}=\frac{2}{3} \sqrt{3} r$. Similarly, the trajectory was sampled

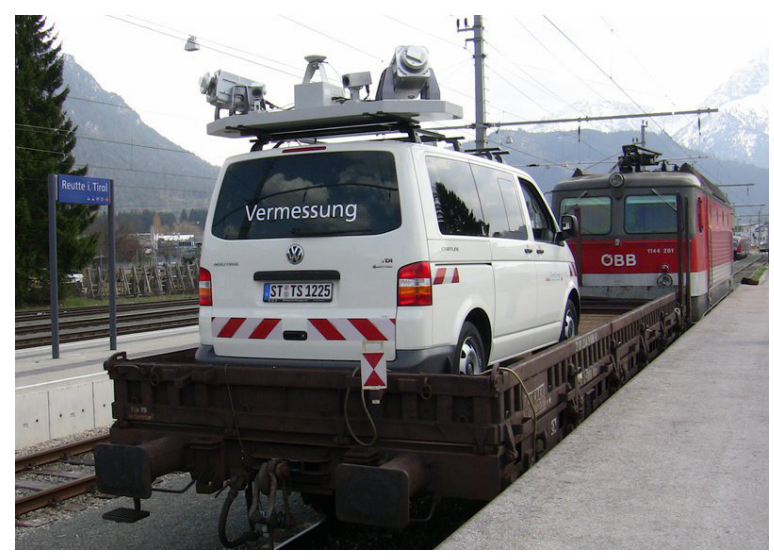

(a) The Optech Lynx Mobile Mapper on the back of a train wagon.

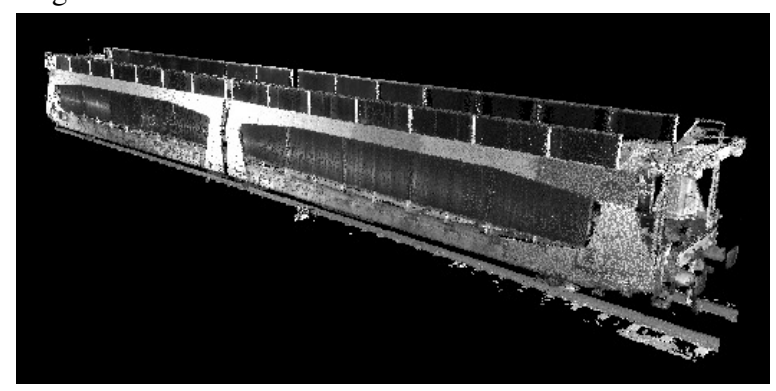

(b) The extracted model of the train wagon.

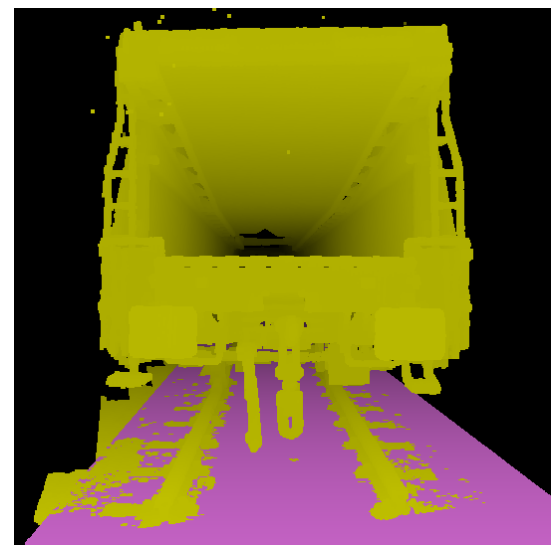

(c) Frontal view of the train wagon and the rectangular base of its bounding box

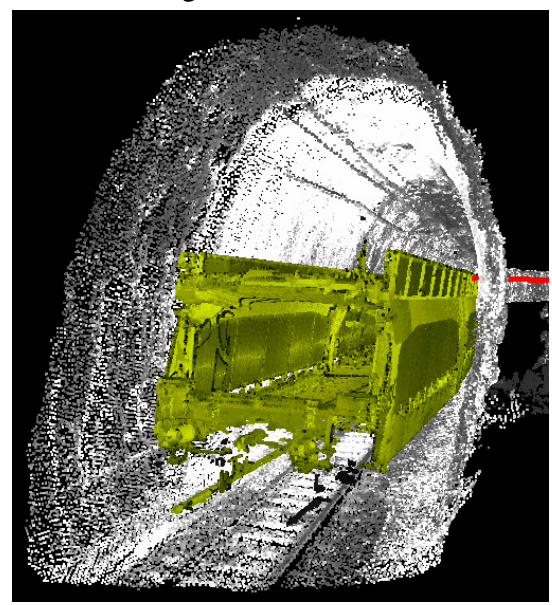

(d) Aligned train wagon (yellow) inside the tunnel environment (gray) and trajectory (red).

Figure 4: The scanning equipment and the scanned wagon. 
Table 1: The first column shows the choice of collision detection search radius $r$. The second column shows the resulting distance between the points of the wagon $d_{m}$ and the points on the trajectory $d_{t}$. The third column shows the resulting number of points in the model. The fourth column shows the resulting number of points on the trajectory. The second and fourth column are extended as the results in figure $5 \mathrm{~b}$ are calculated for higher distance values as well.

\begin{tabular}{cccc}
$r$ in $m$ & $d_{m}=d_{t}=\frac{2}{3} \sqrt{3} r$ & \#model & \#trajectory \\
\hline 0.05 & 0.058 & 28622 & 19392 \\
0.1 & 0.115 & 7546 & 9780 \\
0.2 & 0.231 & 2041 & 4869 \\
0.4 & 0.462 & 461 & 2434 \\
0.8 & 0.924 & 93 & 1217 \\
& 1.848 & & 609 \\
& 3.695 & & 304 \\
& 7.390 & & 152 \\
& 14.780 & & 76
\end{tabular}

such that the individual positions would be between $5.8 \mathrm{~cm}$ and $14.78 \mathrm{~m}$ apart. Table 1 gives an overview of the chosen search radii, the according voxel size and trajectory position distances and the resulting number of points in the model and on the trajectory.

The benchmarks omit runtime results that only modify either the amount of points in the model or the amounts of positions in the trajectory. Both collision detections algorithms scale completely linearly with both variants and can be completely parallelized by splitting the workload over different sets of points in the model or positions in the environment. The benchmarks were done on a Intel Core i5-4200U @ 1.6GHz system with 16GB of system memory and only executed using a single thread.

Figure 5a shows the influence of the search radius on the runtime of both collision detection variants. While all other variables are kept constant, the algorithm was benchmarked with different search radii. The figure shows the runtime of both collision detection variants as well as the number of points that were found to be colliding in each variant. One can observe that the segment based variant finds more colliding points but that it is also slower than the fixed range search based method. Both variants increase exponentially in runtime with higher search radii. With small radii in the centimeter scale, which is desirable for precise results, the runtime of both variants stays below 10 seconds.

In figure $5 b$ the search radius is kept constant and the sampling rate of the trajectory is modified to investigate the dependency of the segment based collision detection method on the segment size. One can observe that as the segment size grows larger, the computation time quickly converges to a constant value of under 10 seconds. The amount of found colliding points slightly increases with larger segment sizes as more colliding points will be found inside the curvature of the tunnel wall.

Figure $5 \mathrm{c}$ shows a more realistic setup in the sense that not only the search radius is modified but also the sampling rate of the trajectory and train wagon model. If the search radius grows, the sampling rates can be lower because more volume is covered. For each value of search radius the sampling rates have been chosen such that no points of the environment would be skipped as the model moves along its trajectory. The graph in figure $5 \mathrm{c}$ shows that the algorithm quickly approaches runtimes below five seconds as the amount of required k-d tree searches decreases with higher search radii and thus lower sampling rates. On the other hand it can be seen that with the lowest and thus most precise search radius of $5 \mathrm{~cm}$ which searches on a trajectory of

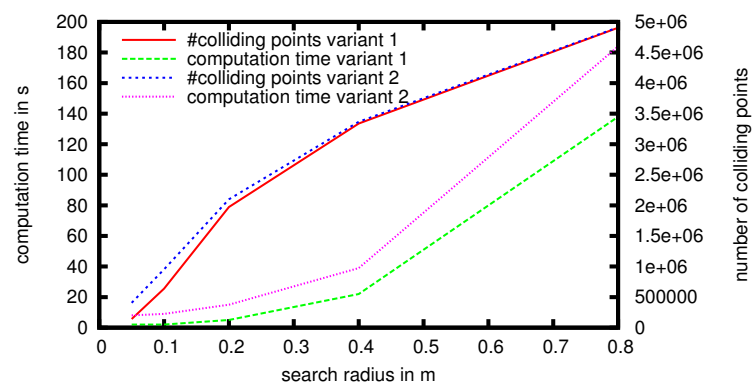

(a) Computation time of both collision detection variants with different search radii $r$. The distance between individual points on the trajectory $d_{t}$ and the distance between points in the model $d_{m}$ is chosen to be $d_{t}=d_{m}=0.231 \mathrm{~m}$.

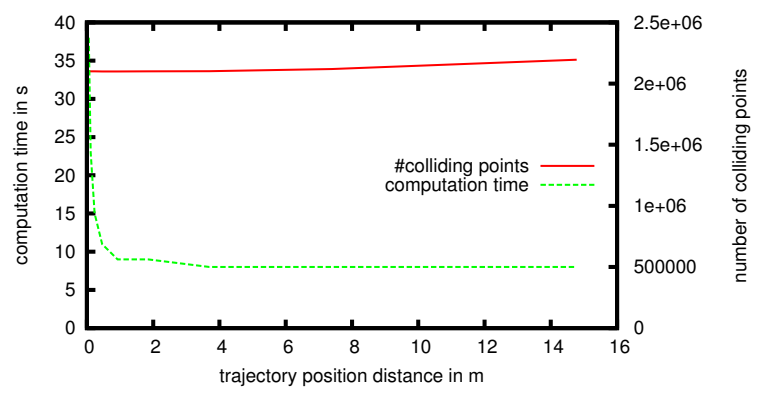

(b) Computation time of the segment based collision detection variant with different distances between individual points on the trajectory with a model sampled with $d_{m}=0.231 \mathrm{~m}$ and a search radius of $0.2 m$.

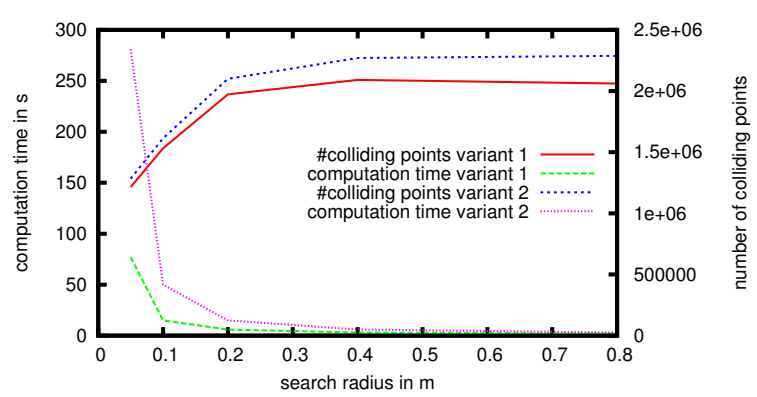

(c) Computation time of both collision detection variants with different search radii $r$. The distance between individual points on the trajectory $d_{t}$ and the distance between points in the model $d_{m}$ is chosen such that $d_{t}=d_{m}=\frac{2}{3} \sqrt{3} r$.

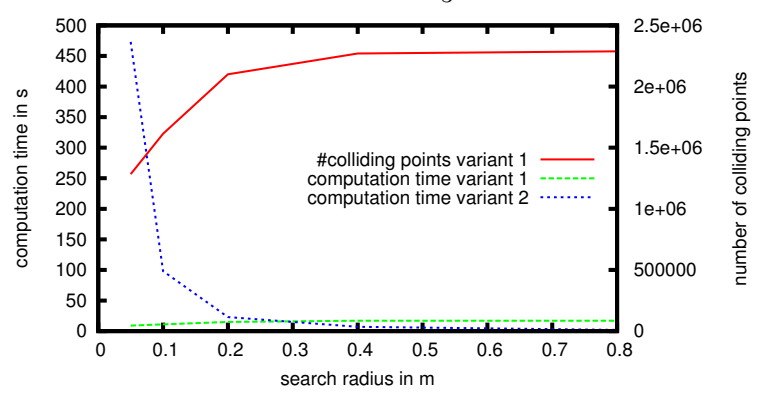

(d) Computation time of both penetration depth variants with different search radii $r$. The distance between individual points on the trajectory $d_{t}$ and the distance between points in the model $d_{m}$ is chosen such that $d_{t}=d_{m}=\frac{2}{3} \sqrt{3} r$. Colliding points were computed using the segmentation based collision detection variant.

Figure 5: Benchmark results 
19,392 positions a model of 28,622 points, our $\mathrm{k}-\mathrm{d}$ tree is able to make all required $19,392 \times 28,622=555,037,824 \mathrm{k}-\mathrm{d}$ tree searches in only 77 seconds. This means that the average k-d tree search in a dataset of 18.92 Mill points takes 139 ns. This in turn means that collision detections of even complex models with up to 287000 points could be done in real time speed of 25.0 frames per second with the presented k-d search tree implementation.

In the last figure $5 \mathrm{~d}$ the two depth of penetration methods are compared. One can see that the first variant stays below $20 \mathrm{sec}-$ onds of computation time in all variants. This is expected as the performance of the algorithm only depends on the amount of colliding points found. We can observe that the first variant increases in runtime slightly as the mount of colliding points rises with increased search radius. The second, more precise depth of penetration variant performs badly for small search radii for which a large number of $\mathrm{k}-\mathrm{d}$ tree searches have to be performed but quickly approaches runtime values below one minute as the search radius grows larger than $10 \mathrm{~cm}$.

\section{CONCLUSIONS AND OUTLOOK}

This paper presented a highly efficient k-d tree implementation which was used to perform collision detection of a sampled arbitrary point cloud against an environment of several million points. It was shown that even though this is a partly brute-force method as it checks all sampled points of the model, it performs well enough such that real queries of densely sampled trajectories can be completed in a matter of seconds. Two heuristics for calculating penetration depth have been presented which work for different scenarios and have different precision and runtime properties. All of these algorithms are embarrassingly parallel and thus speed-ups can easily be gained.

For future work, several routes to improve these methods exist. More work has to be done to research which checks to abort the k$\mathrm{d}$ tree traversal for different search geometries and input data perform best. Another easy way to increase the performance could be to change the sampling of the model from bounding spheres to different geometries like axis aligned bounding boxes which are similarly quick to check for collisions. Lastly, instead of checking every point of the model, a hierarchy of bounding spheres or other geometries could be used (Tzafestas and Coiffet, 1996) but that would destroy the property of the current algorithm that the input model is allowed to arbitrarily deform.

\section{REFERENCES}

Ar, S., Chazelle, B. and Tal, A., 2000. Self-customized bsp trees for collision detection. Computational Geometry 15(1), pp. 91102.

Bergen, G. v. d., 1997. Efficient collision detection of complex deformable models using aabb trees. Journal of Graphics Tools 2(4), pp. 1-13.

Cohen, J. D., Lin, M. C., Manocha, D. and Ponamgi, M., 1995. I-collide: An interactive and exact collision detection system for large-scale environments. In: Proceedings of the 1995 symposium on Interactive 3D graphics, ACM, pp. 189-ff.

Dierckx, P., 1993. Curve and surface fitting with splines. Oxford University Press, Inc.

Elseberg, J., Borrmann, D., Schauer, J., Nüchter, A., Koriath, D. and Rautenberg, U., 2014. A sensor skid for precise 3d modeling of production lines. ISPRS Annals of Photogrammetry, Remote Sensing and Spatial Information Sciences II-5, pp. 117-122.
Garcia-Alonso, A., Serrano, N. and Flaquer, J., 1994. Solving the collision detection problem. Computer Graphics and Applications, IEEE 14(3), pp. 36-43.

Gottschalk, S., Lin, M. C. and Manocha, D., 1996. Obbtree: A hierarchical structure for rapid interference detection. In: Proceedings of the 23rd annual conference on Computer graphics and interactive techniques, ACM, pp. 171-180.

Held, M., Klosowski, J. T. and Mitchell, J. S., 1995. Evaluation of collision detection methods for virtual reality fly-throughs. In: Canadian Conference on Computational Geometry, Citeseer, pp. 205-210.

Hermann, A., Drews, F., Bauer, J., Klemm, S., Roennau, A. and Dillmann, R., 2014. Unified gpu voxel collision detection for mobile manipulation planning. In: Intelligent Robots and Systems (IROS), 2014.

Hubbard, P. M., 1996. Approximating polyhedra with spheres for time-critical collision detection. ACM Transactions on Graphics (TOG) 15(3), pp. 179-210.

Jiménez, P., Thomas, F. and Torras, C., 2001. 3d collision detection: a survey. Computers \& Graphics 25(2), pp. 269-285.

Jung, D. and Gupta, K. K., 1996. Octree-based hierarchical distance maps for collision detection. In: Robotics and Automation, 1996. Proceedings., 1996 IEEE International Conference on, Vol. 1, IEEE, pp. 454-459.

Klein, J. and Zachmann, G., 2004. Point cloud collision detection. In: Computer Graphics Forum, Vol. 23, Wiley Online Library, pp. 567-576.

Klosowski, J. T., Held, M., Mitchell, J. S., Sowizral, H. and Zikan, K., 1998. Efficient collision detection using bounding volume hierarchies of k-dops. Visualization and Computer Graphics, IEEE Transactions on 4(1), pp. 21-36.

Lin, M. and Gottschalk, S., 1998. Collision detection between geometric models: A survey. In: Proc. of IMA conference on mathematics of surfaces, Vol. 1, pp. 602-608.

Luque, R. G., Comba, J. L. and Freitas, C. M., 2005. Broadphase collision detection using semi-adjusting bsp-trees. In: Proceedings of the 2005 symposium on Interactive 3D graphics and games, ACM, pp. 179-186.

Nüchter, A., Elseberg, J., Schneider, P. and Paulus, D., 2010. Study of parameterizations for the rigid body transformations of the scan registration problem. Computer Vision and Image Understanding 114(8), pp. $963-980$.

Siegmann, J., 2011. Lichtraumprofil und fahrzeugbegrenzung im europäischen schienenverkehr. http: //www. forschungsinformationssystem.de/servlet/ is/325031/. [Online; accessed 2014-07-14].

Teschner, M., Heidelberger, B., Müller, M., Pomeranets, D. and Gross, M., 2003. Optimized spatial hashing for collision detection of deformable objects. Technical report, Technical report, Computer Graphics Laboratory, ETH Zurich, Switzerland.

Tzafestas, C. and Coiffet, P., 1996. Real-time collision detection using spherical octrees: virtual reality application. In: Robot and Human Communication, 1996., 5th IEEE International Workshop on, pp. 500-506. 